\title{
Two-point Correlator Fits on HISQ Ensembles
}

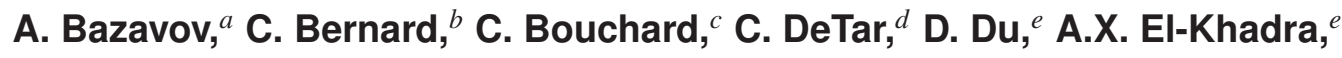
J. Foley, ${ }^{d}$ E.D. Freeland, ${ }^{f}$ E. Gamiz, ${ }^{g}$ Steven Gottlieb, ${ }^{h}$ U.M. Heller, ${ }^{i}$ J.E. Hetrick, ${ }^{j}$ J. Kim ${ }^{*}{ }^{k}$ A.S. Kronfeld, ${ }^{l}$ J. Laiho, ${ }^{m}$ L. Levkova, ${ }^{d}$ M. Lightman, ${ }^{b}$ P.B. Mackenzie, ${ }^{l}$ E.T. Neil, ${ }^{l}$ M. Oktay, ${ }^{d}$ J.N. Simone, ${ }^{l}$ R.L. Sugar,${ }^{n}$ D. Toussaint, ${ }^{k}$ R.S. Van de Water $,{ }^{a},{ }^{l}$ and R. Zhou ${ }^{h}$ [Fermilab Lattice and MILC Collaboration]

${ }^{a}$ Department of Physics, Brookhaven National Laboratory, ${ }^{\dagger}$ Upton, NY 11973, USA

${ }^{b}$ Department of Physics, Washington University, St. Louis, MO 63130, USA

${ }^{c}$ Department of Physics, The Ohio State University, Columbus, OH 43210, USA

${ }^{d}$ Physics Department, University of Utah, Salt Lake City, UT 84112, USA

e Physics Department, University of Illinois, Urbana, IL 61801, USA

${ }^{f}$ Department of Physics, Benedictine University, Listle, IL 60532, USA

${ }^{g}$ CAFPE and Departamento de Fisica Te orica y del Cosmos, Universidad de Granada, Granda, Spain

${ }^{h}$ Department of Physics, Indiana University, Bloomington, IN 47405, USA

${ }^{i}$ American Physical Society, One Research Road, Ridge, NY 11961, USA

j Physics Department, University of the Pacific, Stockton, CA 95211, USA

${ }^{k}$ Physics Department, University of Arizona, Tucson, AZ 85721, USA

${ }^{l}$ Fermi National Accelerator Laboratory, ${ }^{\ddagger}$ Batavia, IL 60510, USA

${ }^{m}$ SUPA, School of Physics and Astronomy, University of Glasgow, Glasgow G12 8QQ, UK

${ }^{n}$ Department of Physics, University of California, Santa Barbara, CA 93106, USA

E-mail: jkimephysics.arizona.edu,

We present our methods to fit the two point correlators for light, strange, and charmed pseudoscalar meson physics with the highly improved staggered quark (HISQ) action. We make use of the least-squares fit including the full covariance matrix of the correlators and including Gaussian constraints on some parameters. We fit the correlators on a variety of the HISQ ensembles. The lattice spacing ranges from $0.15 \mathrm{fm}$ down to $0.06 \mathrm{fm}$. The light sea quark mass ranges from 0.2 times the strange quark mass down to the physical light quark mass. The HISQ ensembles also include lattices with different volumes and with unphysical values of the strange quark mass. We use the results from this work to obtain our preliminary results of $f_{D}, f_{D_{s}}, f_{D_{s}} / f_{D}$, and ratios of quark masses presented in another talk [1].

The 30th International Symposium on Lattice Field Theory

June 24-29, 2012

Cairns, Australia

\footnotetext{
* Speaker.

$\dagger$ Operated by Brookhaven Science Associates, LLC, under Contract No. DE-AC02-98CH10886 with the U.S. Department of Energy.

*Operated by Fermi Research Alliance, LLC, under Contract No. DE-AC02-07CH11359 with the U.S. Department of Energy.
} 


\section{Introduction}

Fitting correlators is one of the essential procedures in studying non-perturbative physics in Lattice QCD. The hadron spectrum and decay constants can be calculated by fitting two-point correlation functions, and hadronic form factor and matrix elements can be calculated by fitting three-point functions. Here we present our method for fitting two-point meson correlators with HISQ.

The meson correlators are computed using the HISQ action[4], which is an $\mathscr{O}\left(a^{2}\right)$-improved staggered quark action, with smaller taste-symmetry breakings than in the asqtad action we used previously. For the pseudoscalar meson correlators, we use both random-wall sources and Coulomb gauge fixed wall sources, with the point operator as the sink. We do combined fits to the correlators of both sources. This helps us to isolate the ground states that we are interested in. More details about the correlators are given in Sec. 2.

The HISQ lattices include the vacuum polarization of four dynamical quarks: up, down, strange, and charm quarks [2,3]. There are, in total, nineteen HISQ ensembles with different lattice spacings, quark masses, and spatial volumes. The parameters of the HISQ lattices are tabulated in Table 1. Note that we have physical sea-quark mass ensembles, where all sea-quark masses are tuned to take the physical values. These ensembles eliminate the need for a chiral extrapolation, and they can be used, together with higher unphysical-mass ensembles, to correct for slight mistunings of the sea quark masses.

Since elements of the correlators at different time slices are highly correlated, we use the full covariance matrix, which includes both diagonal and off-diagonal elements, to define the objective function $\chi^{2}$. We use Gaussian constraints, or priors in the language of Bayesian fits, on some parameters of heavier states. This is useful especially when multiple states are needed to get reliable fits.

We divide the correlators into two groups: light-light and heavy-light. In this terminology, light refers to the up, down, and strange quarks, while heavy refers to the charm quark. We find that we can get good fits with a single state for light-light correlators as long as large enough distances are chosen for the fitting range. However, we find multiple state fits are efficient for heavy-light correlators to get good fits with small statistical errors. Our fitting methods are discussed in more detail in Sec. 3.

We block the correlators in the Monte Carlo trajectory to account for the autocorrelations. To find an optimal size of the blocks, we look at how the covariance matrix scales as the size of the blocks is increased. In the course of this analysis, we find that the autocorrelations depend on (Euclidean) time, and that they can be understood as a consequence of the way the source time slices are chosen. A short discussion about this is given in Sec. 4

The systematic error due to the excited-state contamination is tested by varying the fitting ranges and priors. Changes from these variations are small compared to our statistical errors. This is briefly discussed in Sec. 5 


\section{Meson Correlators}

We use the point and wall operators to create the pseudoscalar mesons,

$$
\begin{aligned}
O_{P}(\vec{x}, t) & =\bar{\chi}_{A}(\vec{x}, t) \varepsilon(\vec{x}, t) \chi_{B}(\vec{x}, t), \\
O_{W}(t) & =\sum_{\vec{x}, \vec{y}} \bar{\chi}_{A}(\vec{x}, t) \varepsilon(\vec{x}+\vec{y}, t) \chi_{B}(\vec{y}, t),
\end{aligned}
$$

where $\chi$ is the HISQ field, and $A, B$ are flavor indices. $\varepsilon(\vec{x}, t)=(-1)^{\sum_{i} x_{i}+t}$ corresponds to the projection to the Goldstone pion with staggered quarks [5]. The Coulomb gauge fixing must be used for the wall operator, so we call this Coulomb wall operator.

Using the point sink, we can define two correlators depending on the source,

$$
\begin{aligned}
C_{R}(t) & =\left\langle\frac{1}{V_{S}} \sum_{\vec{x}} O_{P}(\vec{x}, t) \frac{1}{3 V_{S}} \sum_{\vec{y}} O_{P}^{\dagger}(\vec{y}, 0)\right\rangle, \\
C_{W}(t) & =\left\langle\frac{1}{V_{S}} \sum_{\vec{x}} O_{P}(\vec{x}, t) O_{W}^{\dagger}(0)\right\rangle .
\end{aligned}
$$

The summation over the sources for the point operator is implemented using random noise, so we call $C_{R}$ random wall source correlators.

These correlators can be expressed in terms of masses and amplitudes of relevant excitations. We adopt the following parameterization.

$$
\begin{aligned}
C_{\mathrm{R}}(t) & =\sum_{j=0}^{J-1} A_{j} M_{j}^{3}\left(e^{-M_{j} t}+e^{-M_{j}\left(N_{T}-t\right)}\right)+\sum_{k=0}^{K-1}(-1)^{t} A_{k}^{\prime} M_{k}^{\prime 3}\left(e^{-M_{k}^{\prime} t}+e^{-M_{k}^{\prime}\left(N_{T}-t\right)}\right), \\
C_{\mathrm{W}}(t) & =\sum_{j=0}^{J-1} B_{j} M_{j}^{3}\left(e^{-M_{j} t}+e^{-M_{j}\left(N_{T}-t\right)}\right)+\sum_{k=0}^{K-1}(-1)^{t} B_{k}^{\prime} M_{k}^{\prime 3}\left(e^{-M_{k}^{\prime} t}+e^{-M_{k}^{\prime}\left(N_{T}-t\right)}\right),
\end{aligned}
$$

where $J$ and $K$ are the numbers of ordinary and alternate states respectively. The backward propagations from the image source at $N_{T}$, the temporal size of the lattice, are included because of periodic boundary condition on the mesons.

Following [7, 8], we fit the two correlators simultaneously with common masses. It helps us to isolate the ground states of the point source since the Coulomb wall operator is less contaminated from excited states. Once the masses and amplitudes are fitted, the decay constant is given by [9]

$$
a f_{A B}=\left(a m_{A}+a m_{B}\right) \sqrt{3 V_{S} A_{0} / 2},
$$

where $V_{S}$ is the spatial volume. Note that bare values of masses and amplitude can be used without renormalization thanks to the partially conserved axial current (PCAC) relation of HISQ. Hence, the uncertainty from renormalization is absent.

\section{Least-squares Fits}

We use least-squares minimization to find the best fits. The objective function to be minimized is the augmented $\chi^{2}$ function, which includes the Gaussian priors as well as usual $\chi^{2}$,

$$
\chi_{\text {aug }}^{2}(\theta)=\chi^{2}(\theta)+\sum_{\alpha} \frac{\left(\theta_{\alpha}-\mu_{\alpha}\right)^{2}}{\sigma_{\alpha}^{2}},
$$




\begin{tabular}{|c|c|c|c|c|c|}
\hline$\beta$ & $\approx a(f m)$ & $a m_{l}$ & $a m_{s}$ & $L^{3} \cdot T$ & $N_{\text {lat }}$ \\
\hline \multirow{3}{*}{580} & \multirow{3}{*}{0.15} & 0.013 & 0.065 & $16^{3} \cdot 48$ & 1020 \\
\hline & & 0.0064 & 0.064 & $24^{3} \cdot 48$ & 1000 \\
\hline & & 0.00235 & 0.064 & $32^{3} \cdot 48$ & 1000 \\
\hline \multirow{11}{*}{600} & \multirow{11}{*}{0.12} & 0.0102 & 0.0509 & $24^{3} \cdot 64$ & 1040 \\
\hline & & 0.00507 & 0.0507 & $24^{3} \cdot 64$ & 1020 \\
\hline & & 0.00507 & 0.0507 & $32^{3} \cdot 64$ & 1000 \\
\hline & & 0.00507 & 0.0507 & $40^{3} \cdot 64$ & 1030 \\
\hline & & 0.00184 & 0.0507 & $48^{3} \cdot 64$ & 840 \\
\hline & & 0.00507 & 0.0304 & $32^{3} \cdot 64$ & 1020 \\
\hline & & 0.00507 & 0.022815 & $32^{3} \cdot 64$ & 1020 \\
\hline & & 0.00507 & 0.012675 & $32^{3} \cdot 64$ & 1020 \\
\hline & & 0.00507 & 0.00507 & $32^{3} \cdot 64$ & 1020 \\
\hline & & $0.00507 / 0.012675$ & 0.022815 & $32^{3} \cdot 64$ & 1020 \\
\hline & & 0.0088725 & 0.022815 & $32^{3} \cdot 64$ & 1020 \\
\hline \multirow{3}{*}{630} & \multirow{3}{*}{0.09} & 0.0074 & 0.037 & $32^{3} \cdot 96$ & 1011 \\
\hline & & 0.00363 & 0.0363 & $48^{3} \cdot 96$ & 1000 \\
\hline & & 0.0012 & 0.0363 & $64^{3} \cdot 96$ & 702 \\
\hline \multirow{2}{*}{672} & \multirow{2}{*}{0.06} & 0.0048 & 0.024 & $48^{3} \cdot 144$ & 1000 \\
\hline & & 0.0024 & 0.024 & $64^{3} \cdot 144$ & 655 \\
\hline
\end{tabular}

Table 1: Parameters of HISQ ensembles are tabulated. With a few intentional exceptions, the light quark masses $\left(m_{l}\right)$ are approximately $0.2,0.1$, and 0.037 times the physical strange quark mass. The exceptions appear in the ensembles with unphysical values of the strange quark mass and non-degenerate up and down quark masses. The HISQ ensembles also include three ensembles to study finite-volume effects, where all parameters are the same except the lattice volume.

\begin{tabular}{lllllll}
\hline & \multicolumn{2}{c}{ light-light } & \multicolumn{2}{c}{ heavy-light } \\
\hline No. of states & \multicolumn{2}{c}{$\mathrm{J}+\mathrm{K}=1+0$} & \multicolumn{3}{c}{$\mathrm{J}+\mathrm{K}=2+1$} \\
\hline \multirow{2}{*}{ Priors } & \multicolumn{2}{c}{ No } & & \multicolumn{3}{c}{$M_{1}-M_{0}=700 \pm 140 \mathrm{MeV}$} \\
& & & & $M_{0}^{\prime}-M_{0}=400 \pm 200 \mathrm{MeV}$ \\
\hline \multirow{4}{*}{ Fit range } & 0.15 & 16 & 23 & 0.15 & 8 & 23 \\
& 0.12 & 20 & 31 & 0.12 & 10 & 31 \\
& 0.09 & 30 & 47 & 0.09 & 15 & 47 \\
& 0.06 & 40 & 71 & 0.06 & 20 & 71 \\
\hline
\end{tabular}

Table 2: Our fit methods are summarized. $J+K$ stands for the number of states included in the fits, where $J(K)$ is that of ordinary (alternate) states. For heavy-light, the priors are applied to the mass gaps. The minimum distances are about $2.4 \mathrm{fm}$ for light-light and $1.2 \mathrm{fm}$ for heavy-light. 


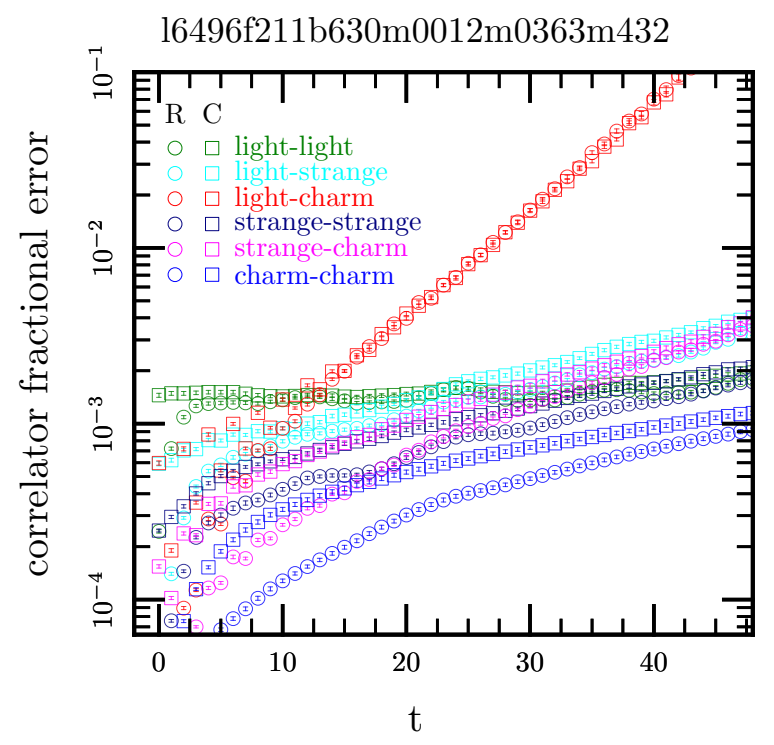

Figure 1: A example of the fractional errors for various valence quark mass combinations are shown. The circle and square symbols stand for random and Coulomb wall sources respectively. Here, "light" stands for up and down quarks.

where $\theta$ represents a set of parameters to be estimated, and $\alpha$ runs over parameters constrained with central value $\mu_{\alpha}$ and width $\sigma_{\alpha}$.

Table 2 summarizes our fit methods. We divide the correlators into two groups depending on the valence quark masses: light-light and heavy-light, where by "heavy" we mean charm and "light", up, down, and strange. For light-light, we use 1+0 state with large minimum distances $(\sim 2.4 \mathrm{fm})$. For heavy-light, however, we use $2+1$ state with constrained masses and the reduced minimum distances $(\sim 1.2 \mathrm{fm})$. We choose minimum distances small enough to provide adequate statistical signal for included states but not so small as to be polluted from ignored excited states.

The main reason for the differences in the two groups of fits is the fractional errors (noiseto-signal) of the correlators [10]. Figure 1 shows an example of the fractional errors for various valence quark combinations. One can easily see that the fractional error grows much faster for heavy-light. This means that good signals are not expected at large distances for heavy-light. Thus, it is better to reduce the minimum distance to get better statistical precision, while including additional heavier states in the fitting function.

The central values of the priors are taken to be $M_{D(2550)}-M_{D} \approx 700 \mathrm{MeV}$ for the ordinary excited state and $M_{D^{*}}-M_{D} \approx 400 \mathrm{MeV}$ for the alternate state [6]. The widths are chosen narrow enough to effectively stabilize the fits, but not so narrow as to significantly influence our determination of the ground states, the states of interest. We find that these priors allow us to get good confidence levels of fits (or $p$-values) over all heavy-light fits. 

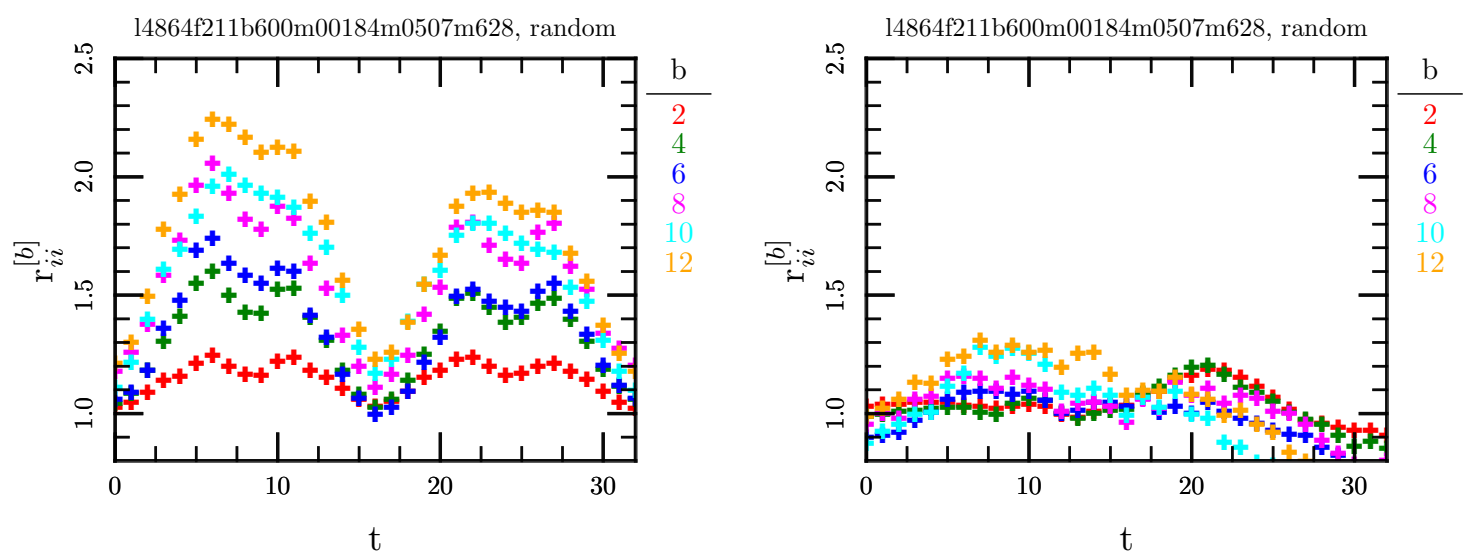

Figure 2: The ratios of diagonal elements of the covariance matrix for the random wall source correlator on the $0.12 \mathrm{fm}$ physical quark mass ensemble are plotted for various values of block size $b$. On the left, the correlators are averaged over four measurements on each lattice. On the right, however, only a single measurement is used to measure the correlators. For details, please see the text.

\section{Autocorrelation of Correlators}

We use the blocking method to compute the correlators and their statistical errors from the Monte Carlo samples. The blocking method divides measurements in a time series into contiguous blocks and uses their means as independent measurements in successive analysis. As a proxy for autocorrelation, we examine ratios of the covariance matrix of the correlators as a function of the block size,

$$
r_{i j}^{[b]} \equiv C_{i j}^{[b]} / C_{i j}^{[1]} .
$$

where $b$ denotes the size of blocks, and $C_{i j}^{[b]}$ represents the covariance matrix computed with the block size $b$.

Figure 2 shows examples of the diagonal elements of the ratio matrix. On the left plot, we can see that the autocorrelation has a peculiar (Euclidean) time dependence. This happens because we measure the correlators from four source times and average them to get one estimator on each gauge configuration. Then, when we proceed to the next gauge configuration, we move the locations of sources by a constant amount $N_{T} / 8$ plus a small displacement $\delta= \pm 1$, which prevents moving back and forth to the same places. Note that the positions of peaks coincide with the "moving distance", $N_{T} / 8+\delta$, or its multiple. We can compare these with the results of the single measurement (with the the moving distance $\sim N_{T} / 4$ ), which are plotted on the right in Fig. 2. Hence, we find these autocorrelations are induced by the way we place the multiple sources thoughout the lattices.

To suppress the effects of the autocorrelations, we need to increase the size of the blocks. However, as the block size increases we have fewer blocks, and our error estimates get less accurate. In this trade-off, we find that an optimal block size is four, which gives us enough statistics 
to estimate the covariance matrices of large dimension while reducing the underestimation of error due to the autocorrelations for the ensembles where the number of configurations is about a thousand. We use the block size two or one depending on the number of configurations when it is less than a thousand.

\section{Excited State Effects}

To address the systematic uncertainty due to ignored excited states, we vary the fitting range and priors and then test changes of the values of interest. For measurements of the decay constants on the $0.09 \mathrm{fm}$ physical sea quark mass ensemble, for example, no statistically significant deviation is found beyond statistical precision with any of the following variations: with reduced prior widths $(\sim 50 \mathrm{MeV})$ for the mass gaps, with shifts of the central values $(\sim \pm 100 \mathrm{MeV})$, with additional constraints on the amplitudes, with changes of the minimum distances $(\sim \pm 2)$, and so on. This leads us to our conservative estimations of the systematic uncertainty from the excited states in Ref. [1].

\section{Acknowledgments}

This work was supported by the U.S. Department of Energy and National Science Foundation. Computation for this work was done at the Texas Advanced Computing Center (TACC), the National Center for Supercomputing Resources (NCSA), the National Institute for Computational Sciences (NICS), the National Center for Atmospheric Research (UCAR), the USQCD facilities at Fermilab, and the National Energy Resources Supercomputing Center (NERSC), under grants from the NSF and DOE.

\section{References}

[1] A. Bazavov et al, (MILC collaboration), PoS (Lattice2012) 159.

[2] A. Bazavov et al. (MILC Collaboration), Phys. Rev. D 82, 074501 (2010) [arXiv:1004.0342 [hep-lat]].

[3] A. Bazavov et al., PoS (Lattice2010) 320.

[4] E. Follana et al. (HPQCD and UKQCD Collaborations), Phys. Rev. D 75, 054502 (2007) [hep-lat/0610092].

[5] M. F. L. Golterman, Nucl. Phys. B 273, 663 (1986).

[6] J. Beringer et al. (Particle Data Group), Phys. Rev. D86, 010001 (2012)

[7] C. Aubin et al. (MILC Collaboration), Phys. Rev. D 70, 114501 (2004) [hep-lat/0407028].

[8] A. Bazavov et al., Rev. Mod. Phys. 82, 1349 (2010) [arXiv:0903.3598 [hep-lat]].

[9] G. W. Kilcup and S. R. Sharpe, Nucl. Phys. B 283, 493 (1987).

[10] G. P. Lepage, CLNS-89-971, C89-06-04 (1989) 